\title{
Viewpoint \\ What does an orphan G-protein-coupled receptor have to do with estrogen?
}

\author{
James M Rae ${ }^{1}$ and Michael D Johnson²
}

\begin{abstract}
${ }^{1}$ Division of Hematology Oncology, Department of Internal Medicine, University of Michigan Medical Center, Ann Arbor, Michigan, USA ${ }^{2}$ Department of Oncology, Georgetown University, Washington, District of Columbia, USA
\end{abstract}

Corresponding author: James M Rae, jimmyrae@umich.edu

Published: 29 September 2005

This article is online at http://breast-cancer-research.com/content/7/6/243

(c) 2005 BioMed Central Ltd

Breast Cancer Research 2005, 7:243-244 (DOI 10.1186/bcr1330)

\begin{abstract}
Estrogen affects multiple aspects of human physiology, including the normal growth and development of female reproductive tissues, bone integrity, cardiovascular and central nervous system functions, and plays a central role in normal mammary development and breast pathogenesis. It modulates diverse cell signaling pathways, some of which appear to be independent of the known estrogen receptors (ERs). Although many of estrogen's actions can be explained by the nuclear ERs (ER- $\alpha$ and ER- $\beta$ ) functioning as ligand-activated RNA transcription factors, there are numerous rapid biochemical and physiological responses that cannot be explained by the classical genomic effects of estrogen signaling. It has long been postulated that the rapid effects of estrogen are due to a membrane-bound ER, and two recent reports suggest that it is in fact a G-protein-coupled receptor named 'GPR30'.
\end{abstract}

In 1977 Pietras and Szego [1] showed that plasma membranes prepared from endometrial and liver cells contain a high-affinity estrogen-binding site, but this work was largely ignored by breast cancer researchers and dismissed as a result of experimental artifact due to contamination of the membrane preparations with estrogen receptor (ER)- $\alpha$. Recent studies examining the identity of the membrane-bound estrogen receptor (mER) have diverged into two schools of thought, the first being that the $m E R$ is in fact the classical nuclear ER- $\alpha$ (and ER- $\beta$ ) relocated to the plasma membrane $[2,3]$; the second proposes that an orphan G-protein-coupled receptor (GPCR) protein 'GPR30' is the mER [4,5].

In the 'classic ER at the cell surface camp', Levin and colleagues (for review, see Evinger and Levin [3]) suggested that the rapid effects of estrogen are mediated by a subpopulation of the nuclear ER- $\alpha$ and ER- $\beta$ proteins, shuttled to the plasma membrane by the scaffold protein caveolin-1, and complexed with multiple signaling molecules, which then functions as an atypical GPCR. Although their data are intriguing and suggest that classical ERs may play a role in cellular signaling outside the nucleus, this model is hard to reconcile with other findings, such as the rapid effects of estrogen on mouse hippocampal neurons from ER-knockout mice.

There is a growing body of evidence suggesting that $m E R$ is distinct from ER- $\alpha$ and ER- $\beta$, culminating in two recent papers showing that an orphan GPCR, expression of which tends to correlate with that of ER- $\alpha$, binds directly to and can be activated by estrogen $[4,5]$. This receptor, called GPR30, may be involved in eliciting some of the effects of $17 \beta$-estradiol that were previously attributed to interactions with ER- $\alpha$ and ER- $\beta$. The GPCRs as a group have perhaps not been given the attention they deserve by many breast cancer researchers because they are mostly viewed as regulators of various ion channels and not directly involved in cellular growth or differentiation. However, recent studies have shown that GPCRs can influence cellular proliferation and do so by transactivating growth factor receptors including epidermal growth factor receptor (EGFR), plateletderived growth factor receptor, and insulin-like growth factor-1 receptor. This highlights the important role that this class of receptor probably plays, both in normal mammary biology and in the etiology and progression of breast cancer. Interestingly, the estrogen-induced transactivation of EGFR that Levin's group attributes to the classical ER- $\alpha$ at the plasma membrane [2] has also been shown to be mediated by GPR30 [6]. Crosstalk between GPR30 and EGFR is due to the rapid proteolytic release of heparin-bound epidermal growth factor by matrix metalloproteases, which allows the ligand to bind to and activate EGFR.

Originally identified in a screen for neurotransmitter receptors in a Burkitt's lymphoma cell line, GPR30 was independently cloned in 1997 by Weigel and colleagues [7], who were conducting a differential screen of an MCF-7 cDNA library in

EGFR $=$ epidermal growth factor receptor; ER $=$ estrogen receptor; GPCR $=$ G-protein-coupled receptor; mER $=$ membrane-bound estrogen receptor. 
an attempt to identify genes overexpressed in ER-positive versus ER-negative breast cancers. They mapped GPR30 to chromosome 7p22; its 2604 base pair transcript contains a 1126 open reading frame that encodes a 375 amino acid protein, expression of which correlates with ER- $\alpha$ in breast cancer cell lines and primary breast carcinomas. GPR30 has significant sequence homology to the angiotensin $\| 1 \mathrm{~A}$, interleukin $8 \mathrm{~A}$, and chemokine type 1 receptors, suggesting that the protein might be the receptor for a peptide or glycoprotein, although no such ligand has been found. Others have shown that GPR30 is expressed in estrogenresponsive tissues including breast, heart, leukocytes, brain, and vascular endothelium (for review, see Filardo [8]).

The involvement of GPR30 in estrogen signaling was first demonstrated by Filardo and coworkers [6] in 2000, when they showed that estrogen-induced Erk-1/2 activation was independent of known estrogen receptors. Although others have demonstrated that this occurs in MCF-7 cells, Filardo and colleagues showed that it also occurs in ER- $\alpha / \beta$ negative, GPR30-positive SKBR3 cells. Furthermore MDAMB-231 cells, which are GPR30-negative due to a genomic deletion, are converted to an estrogen-responsive phenotype by transfection with GPR30. Interestingly, they also showed that the pure antiestrogen $\mathrm{ICl} 182,780$ functions as an agonist toward GPR30.

Although GPR30 was shown to be involved in the rapid nongenomic effects of estrogen, it was not known whether estrogen interacted directly with the GPCR or indirectly through an unknown mER. Two nearly simultaneous reports finally put this to rest, demonstrating that estrogen binds directly to GPR30, making this protein a strong candidate for the mER [4,5]. Thomas and coworkers [5] found that estrogen binds to GPR30 with a $\mathrm{K}_{\mathrm{d}}$ of $2.7 \mathrm{nmol} / \mathrm{l}$, and that it is a displaceable receptor with a single saturable binding site. This work was conducted using the SKBR-3 cell line and an ER- $\alpha / E R-\beta / G P R 30-n e g a t i v e$ embryonic kidney cell line, which only acquires estrogen-binding activity after transfection with a GPR30 expression construct. In addition, they demonstrated that tamoxifen and ICI 182,780 bind to GPR30 and appear to mimic the effects of estrogen. The effects of $\mathrm{ICl} 182,780$ are interesting, given the fact that this compound has always been considered a pure antiestrogen. These recent discoveries, coupled with the known actions of GPR30 and its apparent coexpression in ER- $\alpha$-positive breast cancers and estrogen-responsive tissue, make it an excellent candidate for being the true mER.

The work by Thomas and coworkers was confirmed by Revankar and colleagues [4], who showed that GPR30 binds to estrogen and fluorescent estrogen derivatives, and that this binding results in increased intracellular calcium and phosphatidylinositol 3,4,5-triphosphate. Although these two groups concur on the estrogen-binding characteristics of coworkers believe that GPR30 is located at the plasma membrane, whereas the findings reported by Revanker and colleagues [4], using a fluorescently linked GPR30, suggest that it is located within the endoplasmic reticulum - a conclusion apparently supported by additional studies using a fluorescent marker to locate native GRP30 in several cell lines. These data, which seem to contradict previous studies of GPR30 subcellular distribution, may have added to some of the confusion as to whether GPR30 is indeed the true $\mathrm{mER}$. Nevertheless, the identification, characterization, and demonstration of the estrogen-binding characteristics of GPR30 certainly merit serious consideration by breast cancer researchers that it is a potentially important player in estrogen signaling in the breast.

In conclusion, the literature is replete with studies demonstrating that estrogen can produce a panoply of effects in cells, such as alterations in calcium flux, activation of adenyl cyclase, mitogenactivated protein kinase, among others, that in other systems are seen as a result of the activation of GPRCs. The assumption heretofore has been that these effects were most likely mediated by the canonical ERs (ER- $\alpha$ and ER- $\beta$ ); however, given that in most cases the culture systems used also express GPR30, this assumption needs to be tested. It may be that a better understanding of the role played by GPR30 in global estrogen signaling will provide insight into some of the most challenging and longstanding questions in breast cancer research, such as how estrogen signaling is involved in mammary carcinogenesis, the relationship between estrogen responsive and refractory disease, and the mechanisms by which previously hormone responsive tumors elude that control.

\section{Competing interests}

The author(s) declare that they have no competing interests.

\section{References}

1. Pietras RJ, Szego CM: Specific binding sites for oestrogen at the outer surfaces of isolated endometrial cells. Nature 1977, 265:69-72.

2. Razandi M, Pedram A, Merchenthaler I, Greene GL, Levin ER: Plasma membrane estrogen receptors exist and functions as dimers. Mol Endocrinol 2004, 18:2854-2865.

3. Evinger AJ III, Levin ER: Requirements for estrogen receptor alpha membrane localization and function. Steroids 2005, 70: 361-363.

4. Revankar CM, Cimino DF, Sklar LA, Arterburn JB, Prossnitz ER: A transmembrane intracellular estrogen receptor mediates rapid cell signaling. Science 2005, 307:1625-1630.

5. Thomas P, Pang Y, Filardo EJ, Dong J: Identity of an estrogen membrane receptor coupled to a $\mathrm{G}$ protein in human breast cancer cells. Endocrinology 2005, 146:624-632.

6. Filardo EJ, Quinn JA, Bland KI, Frackelton AR Jr: Estrogen-induced activation of Erk-1 and Erk-2 requires the G protein-coupled receptor homolog, GPR30, and occurs via trans-activation of the epidermal growth factor receptor through release of HBEGF. Mol Endocrinol 2000, 14:1649-1660.

7. Carmeci C, Thompson DA, Ring HZ, Francke U, Weigel RJ: Identification of a gene (GPR30) with homology to the G-proteincoupled receptor superfamily associated with estrogen receptor expression in breast cancer. Genomics 1997, 45:607-617.

8. Filardo EJ: Epidermal growth factor receptor (EGFR) transactivation by estrogen via the G-protein-coupled receptor, GPR30: a novel signaling pathway with potential significance for breast cancer. J Steroid Biochem Mol Biol 2002, 80:231-238. 